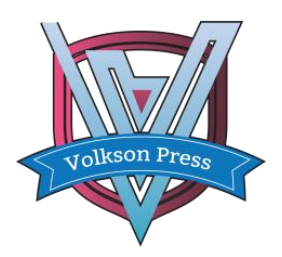

Contents List available at VOLKSON PRESS

Computer Science and Artificial Intelligence(CSAI)

DOI : http://doi.org/10.26480/iscsai.01.2017.40.44

\title{
Research On A Random Forest-Based Underwater Target Image Recognition Method
}

\author{
Min Chen*, Da Song, Weibo Liu, Xiaomei Xie \\ Institute of Astronautics and Aeronautics, University of Electronic Science and "Technology" "Chengdu, China". \\ *Corresponding Author Email: chenmin@uestc.edu.cn
}

This is an open access article distributed under the Creative Commons Attribution License, which permits unrestricted use, distribution, and reproduction in any medium, provided the original work is properly cited

\section{ARTICLE DETAILS}

\section{Article History:}

Received 02 october 2017

Accepted 06 october 2017

Available online 11 october 2017

Keywords:

underwater target, image recognition, random forest, ROC curve

\section{ABSTRACT}

Aimed to solve the problem of the low recognition rate of underwater images in bad underwater environment, an underwater target images' gray level pixel matrix has been employed as the feature matrices to train random forest classifier and get the final classification result from the votes of multi decision trees in this paper. The Gaussian noise with different variances are added into the underwater acoustic image to simulate the different levels of complexity in underwater environment, by which the recognition rate and false alarm rate of diver, fish, propeller, bubble and pipeline are obtained. Furthermore, the performance of this method by ROC curve and AUC are analyzed, and the simulation results show that if the SNR (Signal-to-noise ratio) of underwater target is not less than $2.62 \mathrm{~dB}$ or the PSNR (Peak signal-to-noise ratio) is not less than $12.7 \mathrm{~dB}$, the method proposed in this paper can detect underwater target with weak signal, and achieve more than $90 \%$ of the target recognition rate.

\section{Introduction}

Target recognition has strong practical requirements in the field of underwater exploration. Due to the complexity of the shallow sea topography and high reverberation intensity, the image quality of optical imaging can be affected by weak underwater light and muddy water quality. However, using imaging sonar also faces the effects of low resolution and high noise [1]. The large number of unknown noise in sonar images interfere those target pixels and affect the recognition rate of underwater targets. To improve the recognition rate of underwater acoustic images, on the one hand, the imaging method of image sonar can be improved to enhance the quality of acoustic imaging. On the other hand, the classifiers can be optimized to improve the recognition rate of underwater acoustic images.

The machine learning methods commonly used in underwater target recognition include hidden Markov model, support vector machine, KNearest Neighbor method, principal component analysis and artificial neural network.

In passive sonar target detection and recognition, researchers usually extract acoustic features like Mel-Frequency Cepstral Coefficients(MFCC), Linear Predictive Coding derived Cepstral Coefficient, and so on, after the pretreatment of underwater ship noise [2]. Then they use classifiers including hidden Markov model, support vector machine and K-nearest neighbor method to achieve underwater target recognition and comparative analysis of recognition effect; in literature, they used wavelet transform to extract target features in passive sonar detection and recognition, and then achieved target recognition through multi-layer perceptron and adaptive kernel classifier [3].

Active sonar detection is mainly based on underwater acoustic imaging of the underwater targets. In literature, Korany Noha achieved classification and recognition through Gaussian mixture model(GMM) by extracting MFCC features and wavelet transform features, and he analyzed the recognition results under different signal-noise-ratio using different features [4]. In literature, in order to solve the problem of robustness in underwater noise environment, they proposed a Gammatone filter bank to remove noise, and extract target features by Hilbert-Huang transform [5]. Finally, they used SVM to classify targets. In recent years, due to the rise of deep learning methods, many teams apply it in the classification of underwater targets.

However, since underwater target recognition is often used in ports or military bases, there is a high demand for timely feedback on identification. Many machine learning methods increase the recognition rate while also increasing the complexity of models [6,7]. This will undoubtedly affect the recognition efficiency, and the recognition rate will also be affected by the underwater environment.

Aiming at the problems in underwater target recognition, this paper will use different signal-to-noise ratio underwater target images as a samples, and introduces random forest classifiers to identify the targets. Moreover, the performance of the method will be analyzed by the ROC and the AUV curves $[8,9]$.

\section{PRINCIPLE OF THE METHOD}

Random forest is an ensemble learning method, which makes decision tree as its base learner. It can be used for classification and regression analysis. As random forest is a combination of multiple decision tree based on classifier, using bagging sampling method, it gets better classification result than another single classifier. Random forest can improve the prediction precision without increasing the computational amount significantly, which is insensitive to the effect of multivariate nonlinearity. Its classification results are robust to data loss and imbalance, and can predict the effect of thousands of explanatory variables.

The research ideas of this paper are as follows:

\begin{tabular}{|c|c|c|c|}
$\begin{array}{c}\text { Underwater } \\
\text { acoustic } \\
\text { image } \\
\text { samples }\end{array}$ & $\begin{array}{c}\text { Add } \\
\text { Gaussian noise }\end{array}$ & $\begin{array}{c}\text { Images in } \\
\text { different } \\
\text { underwater } \\
\text { environment }\end{array}$
\end{tabular}$\longrightarrow \begin{gathered}\text { Random } \\
\text { forest } \\
\text { classifier }\end{gathered} \longrightarrow$ ROC\&AUC

Figure 1: Classification method of underwater objects based on random forest

Firstly, Gaussian noise is added to the underwater acoustic image to simulate different underwater acoustic environments. Then, using the supervised learning method of random forest, a random forest classifier is constructed based on the pixel matrix, and the final classification is determined according to the votes of the multiple decision trees. 


\subsection{Random forest}

Decision tree is a unit of random forest. The decision tree (a binary decision tree, for example) is a tree-structured classifier who consists of a root node, an intermediate node, and a leaf node. Given a sample data $x \in X$, and single decision tree $t \in T$, the prediction function $h(x \mid t)$ of decision tree $t$ and the sample data $x$ can be formalized as follows:

$$
\left\{\begin{array}{l}
\text { non - leaf nodes: } h\left(x \mid N\left(\psi, t_{l}, t_{r}\right)\right)=\left\{\begin{array}{l}
h\left(x \mid t_{l}\right), \text { if } \psi(x)=0 \\
h\left(x \mid t_{r}\right), \text { if } \psi(x)=1
\end{array}\right. \\
\text { leaf nodes }: h(x \mid L(\pi))=\pi
\end{array}\right.
$$

Where $\psi(x)$ is the split function of every node in decision trees, which determines the choice of left and right subtrees in the sample data from the root node to the leaf node, and $\pi$ is the category information of leaves. Random forest is a strong classifier composed of a large number of weak classifiers (decision trees). Its classification results are obtained by averaging the results of member decision trees. The classification result of random forest is generally voted by the sub-decision trees, which can be expressed as follows:

$$
y^{*}=\underset{y \in Y}{\arg \max } \sum_{t \in F} I(h(x \mid t)=y)
$$

Where $I(\square)$ is an indicator function? Random forest is to choose the most voted category as the final category of sample data.

Breiman's theoretical research provides a substantial theoretical basis for improving random forest [10]. Suppose the function that generates the $i$ th decision tree is: $f\left(x, \theta_{i}\right): X \longrightarrow Y$, the decision tree is obtained by training samples $x$ and random vectors $\theta$, where $\theta_{i}$ is an independent and uniformly distributed random vector, which can be a random sample with replay of the training samples or a random sample of the number of split nodes in the construction of the decision tree. The forest can now be defined as: $F=\left\{f_{1}, f_{2}, \cdots, f_{T}\right\}$, where $\mathrm{T}$ is the size of the forest.

The margin function of sample $(x, y)$ with labels for a random forest is:

$$
m g(x, y)=a v_{T} I\left(f_{T}(x)=y\right)-\max _{j \neq y} a v_{T} I\left(f_{T}(x)=j\right)
$$

The marginal function describes how well the average of the correct voting categories exceeds that of the other categories. If $m g(x, y)>0$, the random forest gets a correct classification. The marginal value is somewhat similar to the distance from the data point to the classification surface, The larger the value is, the more correct the classification of the forest will be. Therefore, the generalized error of the forest can be defined as:

$$
G E=E_{(X, Y)}(m g(x, y)<0)
$$

Where subscript $(X, Y)$ indicates the entire sample data space and the sample set $\{(X, Y)\}$ is randomly drawn from the entire space $(X, Y)$. The generalization error has an upper bound:

$$
G E^{*} \leq \bar{\rho} \frac{1-s^{2}}{s^{2}}
$$

Where $\bar{\rho}$ is the average correlation between the decision tree under the entire forest, and $S$ is the classification intensity of the decision tree in the forest, which is also the classification accuracy. $S$ is defined as the expectation of the marginal function in the entire sample space, and the formula is as follows:

$$
s=E_{X, Y}\left(P_{\theta}\left(h(X, \theta)=Y-\max P_{\theta}(h(X, \theta)=j)\right)\right.
$$

Where $\bar{\rho}$ can be obtained from the variance of forest marginal function and the average standard deviation of the decision tree is:

$$
\bar{\rho}=\operatorname{var}(m r) /\left(E_{\theta} s d(\theta)\right)^{2}
$$

\subsection{Sample processing}

The sonar images used in this paper are collected from Sound Metrics Didson of Seatronics. Assuming background clutter after the image background clutter is approximately zero-mean Gaussian white noise, the target intensity obeys Gaussian distribution, whose mean is $\mu_{t}$, and variance is $\sigma_{t}^{2} . b$ is a random variable of background noise (including noise), $\mathrm{s}$ is a random variable of target signal strength, and $\mathrm{x}$ is an observation data. Two hypotheses for determining existence are:

$$
\left\{\begin{array}{l}
H_{0}: x=b \quad t \arg \text { ets don't exist } \\
H_{1}: x=s+b \quad t \arg \text { ets exist }
\end{array}\right.
$$

Setting a threshold $T h$, the output signal $\mathrm{U}$ is the threshold function calculated by the image information, that is, $\mathrm{U}$ is the gray scale of the image. $P\left(U \mid H_{0}\right)$ And $P\left(U \mid H_{1}\right)$ are the conditional probability density function of $U$ in the non-objective and objective hypotheses in the image. The false alarm probability is the ratio of the number of times the target is detected but not actually present to the number of detections. Detection probability refers to the ratio of the number of times the target is detected to the number of times the target actually exists.

Given set background clutter $B \in N\left(\mu_{b}, \sigma_{b}^{2}\right)$, target $T \in N\left(\mu_{t}, \sigma_{t}^{2}\right)$, so the probability density function of $P\left(U \mid H_{0}\right)$ and $P\left(U \mid H_{1}\right)$ are:

$$
\begin{aligned}
& P\left(U \mid H_{1}\right)=-\frac{1}{\sqrt{2 \pi \sigma_{t}}} \exp \left[-\frac{\left(x-\mu_{t}\right)}{2 \sigma_{t}^{2}}\right] \\
& P\left(U \mid H_{0}\right)=-\frac{1}{\sqrt{2 \pi \sigma_{b}}} \exp \left[-\frac{\left(x-\mu_{b}\right)}{2 \sigma_{b}^{2}}\right]
\end{aligned}
$$

Probability of Detection $P_{d}$ is the probability of exceeding the detection threshold when both signal and noise exist, and the formula can be expressed as:

$$
\begin{aligned}
P_{d} & =\int_{T h}^{\infty} P\left(U \mid H_{1}\right) d x \\
& =\int_{T h}^{\infty} \frac{1}{\sqrt{2 \pi \sigma_{t}}} \exp \left[-\frac{\left(x-\mu_{t}\right)^{2}}{2 \sigma_{t}^{2}}\right] d x \\
& =1-\Phi\left(\frac{T h-\mu_{t}}{\sigma_{t}}\right) \\
& =\Phi\left(\frac{\mu_{t}-T h}{\sigma_{t}}\right)
\end{aligned}
$$

According to the definition of signal-to-noise ratio:

$S N R=\frac{\mu_{t}-\mu_{b}}{\sigma_{b}}$

Then:

$$
\mu_{t}=S N R \cdot \sigma_{b}+\mu_{b}
$$

Substituting equation (2.5) and equation (2.6) into equation (2.4), we obtain:

$$
P_{d}=\Phi\left(\frac{S N R \cdot \sigma_{b}+\mu_{b}-T h}{\sigma_{t}}\right)
$$

Equation 2.7 shows that the detection probability increases with the increase of signal-to-noise ratio when the detection threshold is 
constant.

\section{SIMULATION ANALYSIS}

\subsection{Sample Processing}

The target recognition probability and the false alarm probability are the two most important evaluation indexes in target detection and recognition system. The relationship between detection probability, false alarm probability and signal-to-noise ratio (SNR) can be obtained by the receiver operating characteristic (ROC) curve. In active sonar signal detection, the main background noise is reverberation. When the signal to noise ratio is constant, the smaller the probability of false alarm is, the greater the detection probability will be.

In the simulation experiment, in order to simulate the underwater acoustic environment of different waters, Gaussian noise with different intensities and Rayleigh noise are added in the original acoustic images, which compose the underwater acoustic image set. The detection probability and false alarm probabilities of different SNR are obtained by Monte Carlo method, and the ROC curve is plotted. The steps are as follows:

(1) According to the given false alarm probability Pf and Monte Carlo number $\mathrm{M}$, the threshold value of $T h$ can be obtained. Firstly, the maximum value of each filter is obtained by inputting the noise into the system M times. After M times, the maximum value is sorted in descending order. The desired threshold $T h$ is the first (Pf* M) order of test after sorting.

(2) According to the given signal-noise ratio SNR and the threshold $T h$, the detection probability $P_{d}$ can be obtained. The signal (including the useful signal and noise) has been inputted into the system M times. After the signal is filtered, the maximum filtered signal is compared with the threshold Th. If the output maximum value is greater than the threshold value $T h$, it can be determined that the signal is detected; on the other hand, if the output maximum value is smaller than the threshold value $T h$, it can be determined that no signal is detected. Then, the number of times that $\mathrm{m}$ is greater than the threshold $T h$ will be counted, and the detection probability $P_{d}$ is equal to $\mathrm{m} / \mathrm{M}$.

In image recognition, a random forest is used to identify and classify targets. Based on the videos of underwater fishes, divers, bubbles, pipeline and propeller, Monte Carlo method is introduced to add the noise of different peak signal-to-noise ratio to the extracted sequence of images. The random forest model is trained by a large number of samples. Figure 2 (a)-(e) show the target images extracted from the measured video, and (f)-(j) show the images, to which the noise has been added.

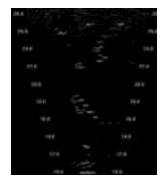

(a)

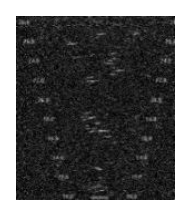

(f)

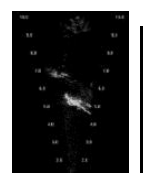

(b)

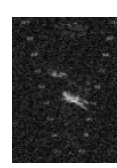

(g)

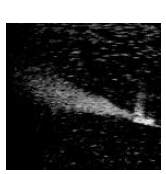

(c)

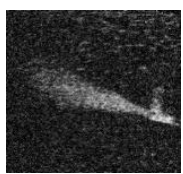

(h)

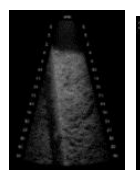

(d)

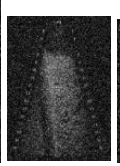

(i)

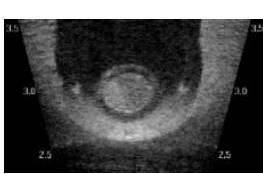

(e)

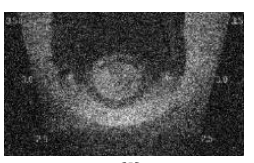

(j)
Figure 2: Underwater target images

\subsection{Experimental Analysis}

A. Random Forest Identification Results Analysis

Using the underwater acoustic image processed in the previous section, the Gaussian variance increases from 0.05 to 20 to simulate the result of underwater target recognition based on random forest in different underwater acoustic environments. The results are shown as follows:

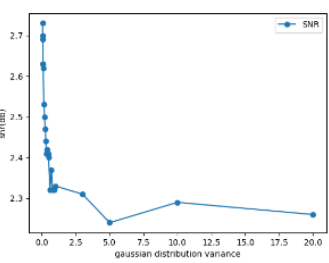

(a)

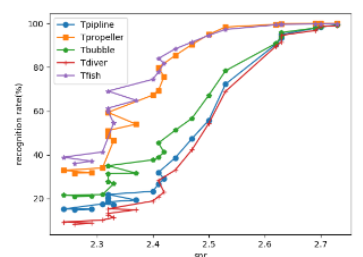

(b)

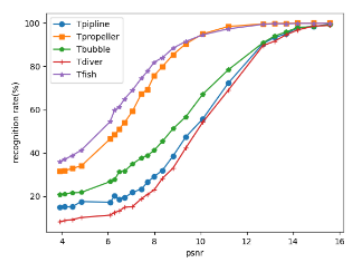

(e)

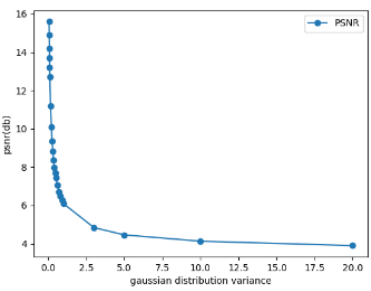

(b)

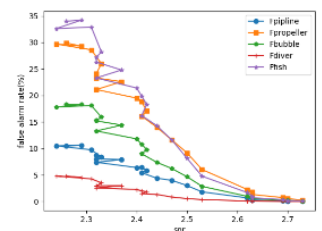

(d)

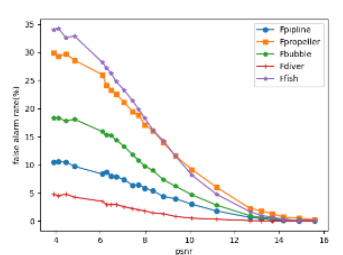

(f)
Figure 3: Target Recognition Rate and False Alarm Rate with Different Signal-to-Noise Ratio

As shown in Figure 3 (a) and (b), the signal-to-noise ratio and peak signalto-noise ratio of the target image are reduced with the increase of Gaussian variance. Figure 3 (c) and (d) show that with the increase of signal-tonoise ratio, the recognition rate also gradually increases while the false alarm rate gradually decreases. Figure 3 (e) and (f) show that with the increase of the peak signal-to-noise ratio, recognition rate gradually increases while the false alarm rate gradually decreases.

The specific recognition rate and false alarm rate are shown in Table 1: Table 1: Recognition Rates at Different SNRs

\begin{tabular}{|c|c|c|c|c|c|c|c|c|}
\hline & 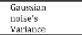 & SNRUWE? & msvguası & Dipelatiae & propentle: & moliber & wiver & Whates \\
\hline 0.05 & 2,35 & 15.6 & 92:48. & too: & 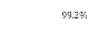 & 9095 & 39.9 & \\
\hline$w .06$ & 27 & 14,9 & $9855 \times$ & 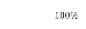 & 90,45 & 9860 & $9,9,8$ & \\
\hline$\omega, w z$ & $20+4$ & 162 & 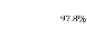 & tan: & 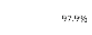 & Fhex:t & - & \\
\hline ana & 2,13 & $12, \overline{7}$ & $95.5 \times$ & $9.9 \%$ & $9 r .96 i$ & 9164 & 99.1. & \\
\hline 0.03 & 2.53 & 13.2 & 9336 & $99.7 \%$ & 9.10\%: & 91658 & 399 & \\
\hline 0.1 & 2.62 & 127 & $90.5 \mathrm{~W}$ & 93.65 & $50.9 \%$ & 87685 & 92,4 & \\
\hline w.15 & 2.58 & 11.2 & 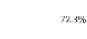 & 0.4 .45 & ro.4.45 & mewatit & ${ }_{47,3}$ & \\
\hline 2.2 & 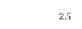 & 19.1. & $\sin$ & 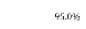 & $6.22 \%$ & 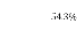 & stik & \\
\hline 2.25 & $2: 7$ & 936 & 17.14 & $90,4 s_{5}$ & s.,.74 & 1234 & 91.5 & \\
\hline 0.3 & 244 & 881 & 38.55 & $85,4=$ & $\$ 12 \%$ & $3300 \mathrm{~B}$ & ва. & \\
\hline 0,255 & ${ }_{341}$ & ${ }_{835}$ & $32,0.5$, & 79,95 & 45,45 & 2835 & $8+0$, & \\
\hline
\end{tabular}

Table 2: False alarm rates under different SNRs

\begin{tabular}{cccccccc}
\hline $\begin{array}{c}\text { Gaussian } \\
\text { noise's } \\
\text { Variance }\end{array}$ & $\begin{array}{c}\text { SNR } \\
(\mathrm{dB})\end{array}$ & $\begin{array}{c}\text { PSNR } \\
(\mathrm{dB})\end{array}$ & pipeline & propeller & bubble & diver & fishes \\
\hline 0.05 & 2.73 & 15.6 & $0.0455 \%$ & $0.241 \%$ & $0.0743 \%$ & 0 & $0.145 \%$ \\
0.06 & 2.7 & 14.9 & $0.0556 \%$ & $0.524 \%$ & $0.200 \%$ & 0 & $0.179 \%$ \\
0.07 & 2.69 & 14.2 & $0.116 \%$ & $0.723 \%$ & $0.274 \%$ & 0 & $0.338 \%$ \\
0.08 & 2.63 & 13.7 & $0.247 \%$ & $1.31 \%$ & $0.509 \%$ & $0.0459 \%$ & $0.690 \%$ \\
0.09 & 2.63 & 13.2 & $0.450 \%$ & $1.83 \%$ & $0.634 \%$ & $0.0765 \%$ & $1.01 \%$ \\
0.1 & 2.62 & 12.7 & $0.662 \%$ & $2.25 \%$ & $0.966 \%$ & $0.0816 \%$ & $1.67 \%$ \\
0.15 & 2.53 & 11.2 & $1.81 \%$ & $6.03 \%$ & $2.85 \%$ & $0.347 \%$ & $4.77 \%$ \\
0.2 & 2.5 & 10.1 & $3.00 \%$ & $9.18 \%$ & $4.67 \%$ & $0.526 \%$ & $8.16 \%$ \\
0.25 & 2.47 & 9.36 & $3.98 \%$ & $11.6 \%$ & $6.21 \%$ & $0.827 \%$ & $11.6 \%$ \\
0.3 & 2.44 & 8.81 & $4.39 \%$ & $14.0 \%$ & $7.38 \%$ & $1.33 \%$ & $14.3 \%$ \\
0.35 & 2.41 & 8.35 & $5.42 \%$ & $16.1 \%$ & $9.00 \%$ & $1.45 \%$ & $16.2 \%$ \\
\hline
\end{tabular}


As shown above, When SNR is higher than $2.6 \mathrm{~dB}$, for all targets, the recognition rate can reach more than $89 \%$, and the false alarm rate is lower than 2.3\%; when the PSNR is greater than $12.7 \mathrm{~dB}$, the recognition rate is more than $89 \%$ for all targets, and the false alarm rate drops to $2.3 \%$ or lower.

Table 1 and Table 2 show the classification of each target using the random forest method. For the fish, the detection probability can reach more than $85 \%$ when the PSNR reaches $8.4 \mathrm{~dB}$ or above; for the frogman target, the detection probability can reach more than $85 \%$ when the PSNR reaches $12.3 \mathrm{~dB}$ or above; Under the condition of underwater bubble target, the detection probability can reach more than $85 \%$ when the PSNR is above $12.0 \mathrm{~dB}$; for the underwater pipeline target, the detection probability can reach above $85 \%$ when the PSNR reaches $12.3 \mathrm{~dB}$ or above; For underwater propeller targets, the detection probability can reach more than $85 \%$ when the PSNR is above $8.8 \mathrm{~dB}$. The ROC curves are plotted and the AUV is calculated by using the recognition possibility of the targets mentioned above.

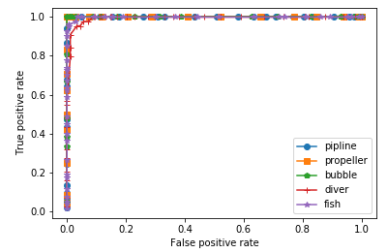

(a) $($ variance $=0.2)$

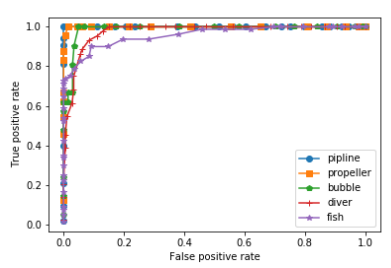

(c) $($ variance $=0.3)$

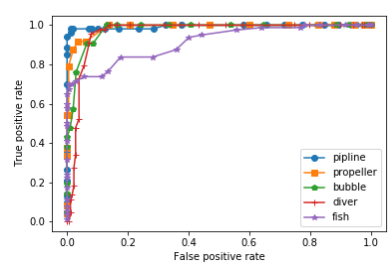

(e) $($ variance $=0.4)$

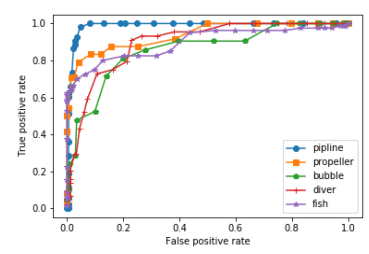

(g) $($ variance $=0.5)$

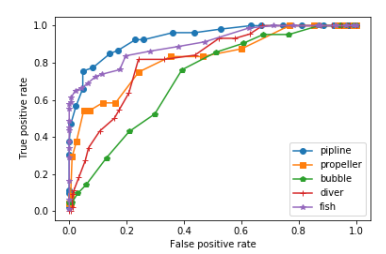

(i) $($ variance $=0.7)$

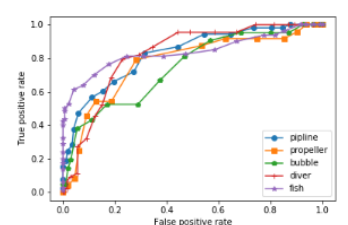

(k) $($ variance $=0.9)$

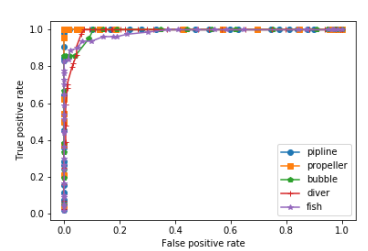

(b) $($ variance $=0.25)$

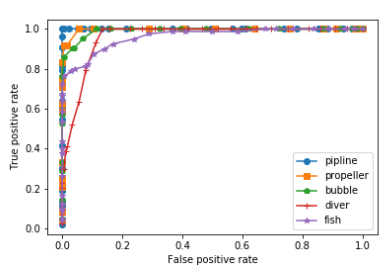

(d) $($ variance $=\mathbf{0 . 3 5})$

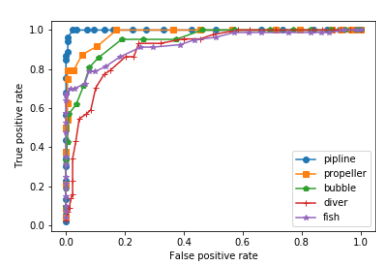

(f) $($ variance $=0.45)$

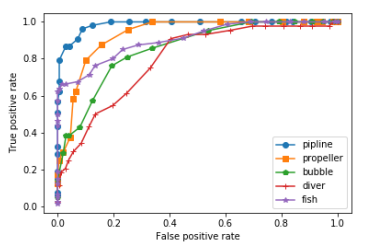

(h) $($ variance $=0.6)$

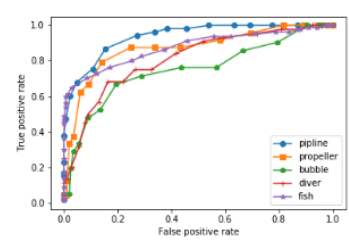

(j) $($ variance $=0.8)$

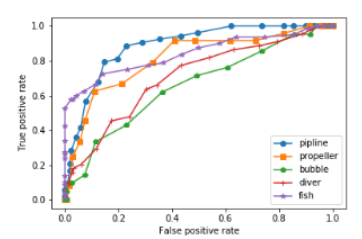

(l) $($ variance $=1.0)$

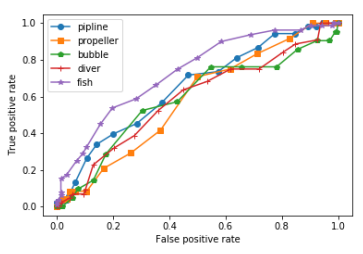

$(\mathrm{m})($ variance $=3.0)$

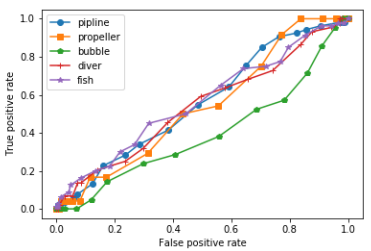

(o) $($ variance $=\mathbf{1 0 . 0})$

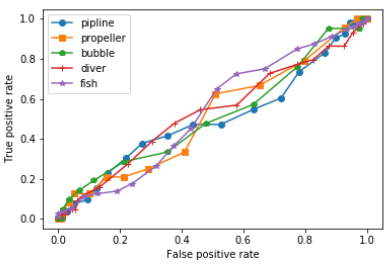

(n) $($ variance $=5.0)$

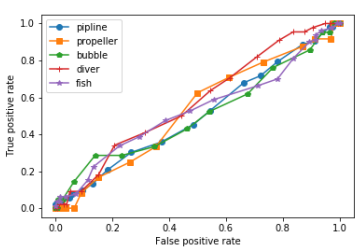

(p) $($ variance $=\mathbf{2 0 . 0})$
Figure 4: ROC curves of all kinds of targets based on random forest Figure 4 shows that as the variance of noise increases from 0.2 to 20.0 , the ROC curves of all targets are getting closer to the lower right corner from the upper left corner. The specific AUC is shown as below:

Table 3: AUC of all targets in different noise variance

\begin{tabular}{llllll}
\hline $\begin{array}{l}\text { Noise } \\
\text { variance }\end{array}$ & $\begin{array}{l}\text { Pipeline } \\
\text { AUC }\end{array}$ & $\begin{array}{l}\text { Propeller } \\
\text { AUC }\end{array}$ & $\begin{array}{r}\text { Bubble } \\
\text { AUC }\end{array}$ & $\begin{array}{l}\text { Diver } \\
\text { AUC }\end{array}$ & $\begin{array}{l}\text { Fish } \\
\text { AUC }\end{array}$ \\
\hline 0.2 & 1.0 & 1.0 & 1.0 & 0.99 & 0.99 \\
0.25 & 0.99 & 1.0 & 0.99 & 0.99 & 0.98 \\
0.3 & 1.0 & 0.99 & 0.99 & 0.97 & 0.96 \\
0.35 & 1.0 & 0.99 & 0.99 & 0.96 & 0.96 \\
0.4 & 0.99 & 0.99 & 0.98 & 0.96 & 0.91 \\
0.45 & 0.99 & 0.98 & 0.95 & 0.90 & 0.93 \\
0.5 & 0.99 & 0.94 & 0.86 & 0.90 & 0.90 \\
0.6 & 0.99 & 0.93 & 0.85 & 0.79 & 0.90 \\
0.7 & 0.93 & 0.82 & 0.71 & 0.81 & 0.90 \\
0.8 & 0.93 & 0.87 & 0.75 & 0.81 & 0.88 \\
0.9 & 0.83 & 0.77 & 0.74 & 0.82 & 0.83 \\
1.0 & 0.89 & 0.81 & 0.66 & 0.70 & 0.84 \\
3.0 & 0.65 & 0.58 & 0.59 & 0.59 & 0.73 \\
5.0 & 0.50 & 0.51 & 0.51 & 0.53 & 0.53 \\
10.0 & 0.56 & 0.54 & 0.39 & 0.54 & 0.56 \\
20.0 & 0.50 & 0.52 & 0.50 & 0.58 & 0.53 \\
\hline
\end{tabular}

As shown above, when noise variance is smaller than 0.2, the AUC of all targets are close to 1 . When the variance increases, AUC decreases gradually. In addition, based on the active sonar equation, a preliminary target detection and estimation is performed. Assuming that the sound source level (SL) is $220 \mathrm{~dB}$, the frogman target intensity TS is $-20 \mathrm{~dB}$, the receiving directivity coefficient $\mathrm{DI}$ is $21 \mathrm{~dB}$, the shallow sea noise level NL at $80 \mathrm{kHz} \sim 120 \mathrm{kHz}$ is about $85 \mathrm{~dB}$, The acoustic propagation loss (2TL) is calculated according to different distances. At this time, the echo signal intensities (echo levels) EL at $10 \mathrm{~m}, 50 \mathrm{~m}, 100 \mathrm{~m}, 300 \mathrm{~m}$ and $500 \mathrm{~m}$ are $169.2 \mathrm{~dB}, 145 \mathrm{~dB}, 132 \mathrm{~dB}, 102 \mathrm{~dB}$ and $79 \mathrm{~dB}$ respectively. Recognition rate can reach a higher level.

\section{CONCLUSION}

In this paper, the underwater acoustic environment is simulated by adding random noise in underwater acoustic images. In this process, the random forest classification method is used to replace the artificial extraction of the target feature. After that, the recognition results of different SNRs are analyzed by ROC curve. If the sound source level is above $200 \mathrm{~dB}$, the experiment shows that the target $500 \mathrm{~m}$ away from sonar can be detected. In addition, the above conclusions are estimated under ideal conditions. Under the actual ocean conditions, the target recognition rate will be lower than the above estimate result because of the influence of noise distribution, multipath interference, sound velocity profile, uncertain noise hypothesis, diving posture and other factors.

\section{ACKNOWLEDGMENTS}

We thank the Dr. Feibiao Dong and other experts and scholars who offered help to this paper.

\section{REFERENCES}

[1] Hsing, C., Lee, C.J.D., Chi, M., Lin. 1998. Classification of Underwater Signals Using Wavelet Transforms and Neural Networks. Mathematical and Computer Modelling, 27 (2), 47-60. 
[2] Ferguson, E.L., Ramakrishnan, R., Williams, S.B., Jin, C.T. 2017. Convolutional Neural Networks for Passive Monitoring of a Shallow Water Environment using a Single Sensor. Acoustics, Speech and Signal Processing (ICASSP), IEEE International Conference on.

[3] Qin, H., Li, X., Yang, Z., Shang, M. 2015. When Underwater Imagery Analysis Meets Deep Learning: a Solution at the Age of Big Visual Data. OCEANS'15 MTS/IEEE Washington. IEEE.

[4] Kim, J., Cho, H., Pyo, J., Kim, J., Yu, S.C. 2016. The convolution neural network based agent vehicle detection using forward-looking sonar image. OCEANS 2016 MTS/IEEE Monterey 10.1109/OCEANS.2016.7761209.
[5] Küçükbayrak, M., Güneș, O., Arica, N. 2009. Underwater Acoustic Signal Recognition Methods. Journal of Naval Science and Engineering, 5 (3), 64-78.

[6] Kamal, S., Mohammed, S.C., Pillai, P.R.S., Supriya, M.H. 2013 Deep Learning Architectures for Underwater Target Recognition. Ocean Electronics (SYMPOL), IEEE.

[7] Zeng, X., Wang, S. 2014. Underwater sound classification based on Gammatone filter bank and Hilbert-Huang transform. Signal Processing, Communications and Computing (ICSPCC), 2014 IEEE International Conference on.

[8] Cao, X., Zhang, X., Yu, Y., Niu, L. 2016. Deep Learning-Based Recognition of Underwater Target. Digital Signal Processing (DSP), 2016 IEEE International Conference on 